\title{
COMPACT GRADIENT $\rho$-EINSTEIN SOLITON IS ISOMETRIC TO THE EUCLIDEAN SPHERE
}

\author{
ABSOS ALI SHAIKH ${ }^{1}$, CHANDAN KUMAR MONDAL $^{2}$, PROSENJIT MANDAL ${ }^{3}$ \\ ABSTRACT. In this paper we have investigated some aspects of gradient $\rho$-Einstein Ricci soliton \\ in a complete Riemannian manifold. First, we have proved that the compact gradient $\rho$-Einstein \\ soliton is isometric to the Euclidean sphere by showing that the scalar curvature becomes \\ constant. Second, we have showed that in a non-compact gradient $\rho$-Einstein soliton satisfying \\ some integral condition, the scalar curvature vanishes.
}

\section{INTRODUCTION AND PRELIMINARIES}

A 1-parameter family of metrics $\{g(t)\}$ on a Riemannian manifold $M$, defined on some time interval $I \subset \mathbb{R}$ is said to satisfy Ricci flow if it satisfies

$$
\frac{\partial}{\partial t} g_{i j}=-2 R_{i j}
$$

where $R_{i j}$ is the Ricci curvature with respect to the metric $g_{i j}$. Hamilton [9] proved that for any smooth initial metric $g(0)=g_{0}$ on a closed manifold, there exists a unique solution $g(t)$, $t \in[0, \epsilon)$, to the Ricci flow equation for some $\epsilon>0$. A solution $g(t)$ of the Ricci flow of the form

$$
g(t)=\sigma(t) \varphi(t)^{*} g(0)
$$

where $\sigma: \mathbb{R} \rightarrow \mathbb{R}$ is a positive function and $\varphi(t): M \rightarrow M$ is a 1-parameter family of diffeomorphisms, is called a Ricci soliton. It is known that if the initial metric $g_{0}$ satisfies the equation

$$
\operatorname{Ric}\left(g_{0}\right)+\frac{1}{2} £_{X} g_{0}=\lambda g_{0},
$$

where $\lambda$ is a constant and $X$ is a smooth vector field on $M$, then the manifold $M$ admits Ricci soliton. Therefore, the equation (11), in general, is known as Ricci soliton. If $X$ is the gradient of some smooth function, then it is called gradient Ricci soliton. For more results of Ricci soliton see [2, 7, 8]. In 1979, Bourguignon [1] introduced the notion of Ricci-Bourguignon flow, where

2020 Mathematics Subject Classification: 53C20; 53C21; 53C44.

Key words and phrases: Gradient $\rho$-Einstein Ricci soliton; scalar curvature; Riemannian manifold. 
the metrics $g(t)$ is evolving according to the flow equation

$$
\frac{\partial}{\partial t} g_{i j}=-2 R_{i j}+2 \rho R g_{i j}
$$

where $\rho$ is a non-zero scalar constant and $R$ is the scalar curvature of the metric $g(t)$. Following the Ricci soliton, Catino and Mazzier [4] gave the definition of gradient $\rho$-Einstein soliton, which is the self-similar solution of Ricci-Bourguignon flow. This soliton is also called gradient RicciBourguignon soliton by some authors.

Definition 1.1. [4] Let $(M, g)$ be a Riemannian manifold of dimension $n,(n \geq 3)$, and let $\rho \in \mathbb{R}, \rho \neq 0$. Then $M$ is called gradient $\rho$-Einstein soliton, denoted by $(M, g, f, \rho)$, if there is a smooth function $f: M \rightarrow \mathbb{R}$ such that

$$
R i c+\nabla^{2} f=\lambda g+\rho R g
$$

for some constant $\lambda$.

The soliton is trivial if $\nabla f$ is a parallel vector field. The function $f$ is known as $\rho$-Einstein potential function. If $\lambda>0$ (resp. $=0,<0)$, then the gradient $\rho$-Einstein soliton $(M, g, f, \rho)$ is said to be shrinking (resp. steady or expanding). On the other hand, the $\rho$-Einstein soliton is called gradient Einstein soliton, gradient traceless Ricci soliton or gradient Schouten soliton if $\rho=1 / 2,1 / n$ or $1 / 2(n-1)$. Later, this notion has been generalized in various directions such as $m$-quasi Einstein manifold [11], $(m, \rho)$-quasi Einstein manifold [12], Ricci-Bourguignon almost soliton [13].

Catino and Mazzier [4] showed that compact gradient Einstein, Schouten or traceless Ricci soliton is trivial. They classified three-dimensional gradient shrinking Schouten soliton and proved that it is isometric to a finite quotient of either $\mathbb{S}^{3}$ or $\mathbb{R}^{3}$ or $\mathbb{R} \times \mathbb{S}^{2}$. Huang [10] deduced a sufficient condition for the compact gradient shrinking $\rho$-Einstein soliton to be isometric to a quotient of the round sphere $\mathbb{S}^{n}$.

Theorem 1.1. [10] Let $(M, g, f, \rho)$ be an $n$-dimensional $(4 \leq n \leq 5)$ compact gradient shrinking $\rho$-Einstein soliton with $\rho<0$. If the following condition holds

$$
\begin{aligned}
\left(\int_{M}\left|W+\frac{\sqrt{2}}{\sqrt{n}(n-2)} Z \bowtie g\right|^{2}\right)^{\frac{2}{n}} & +\sqrt{\frac{(n-4)^{2}(n-1)}{8(n-2)}} \lambda \operatorname{vol}(M)^{\frac{2}{n}} \\
& \leq \sqrt{\frac{n-2}{32(n-1)} Y(M,[g])}
\end{aligned}
$$


where $Z=R i c-\frac{R}{n} g$ is the trace-less Ricci tensor, $W$ is the Weyl tensor and $Y(M,[g])$ is the Yamabe invariant associated to $(M, g)$, then $M$ is isometric to a quotient of the round sphere $\mathbb{S}^{n}$.

In 2019, Mondal and Shaikh [14] proved the isometry theorem for gradient $\rho$-Einstein soliton in case of conformal vector field. In particular, they proved the following result:

Theorem 1.2. [14 Let $(M, g, f, \rho)$ be a compact gradient $\rho$-Einstein soliton. If $\nabla f$ is a nontrivial conformal vector field, then $M$ is isometric to the Euclidean sphere $\mathbb{S}^{n}$.

Dwivedi [13] proved an isometry theorem for gradient Ricci-Bourguignon soliton.

Theorem 1.3. [13] A non-trivial compact gradient Ricci-Bourguignon soliton is isometric to an Euclidean sphere if any one of the following holds

(1) $M$ has constant scalar curvature.

(2) $\int_{M} g(\nabla R, \nabla f) \leq 0$.

(3) $M$ is a homogeneous manifold.

We note that Catino et. al. [5] proved many results for gradient $\rho$-Einstein soliton in noncompact manifold.

Theorem 1.4. Let $(M, g, f, \rho)$ be a complete non-compact gradient shrinking $\rho$-Einstein soliton with $0<\rho<1 / 2(n-1)$ bounded curvature, non-negative radial sectional curvature, and nonnegative Ricci curvature. Then the scalar curvature is constant.

In this paper, we have showed that a non-trivial compact gradient $\rho$-Einstein soliton is isometric to an Euclidean sphere. The main results of this paper are as follows:

Theorem 1.5. A nontrivial compact gradient $\rho$-Einstein soliton has constant scalar curvature and therefore $M$ is isometric to an Euclidean sphere.

We have also showed that in a non-compact gradient $\rho$-Einstein soliton satisfying some conditions the scalar curvature vanishes.

Theorem 1.6. Suppose $(M, g, f, \rho)$ is a non-compact gradient non-expanding $\rho$-Einstein soliton with non-negative scalar curvature. If $\rho>1 / n$ and the $\rho$-Einstein potential function satisfies

$$
\int_{M-B(p, r)} d(x, p)^{-2} f<\infty,
$$

then the scalar curvature vanishes in $M$. 


\section{Proof of the Results}

Proof of the Theorem 1.5. Since the gradient $\rho$-Einstein soliton is non-trivial, it follows that $\rho \neq 1 / n$, see [4]. Taking the trace of (2) we get

$$
R+\Delta f=\lambda n+\rho R n
$$

From the commutative equation, we obtain

$$
\Delta \nabla_{i} f=\nabla_{i} \Delta f+R_{i j} \nabla_{j} f
$$

By using contracted second Bianchi identity, we have

$$
\begin{aligned}
\Delta \nabla_{i} f=\nabla_{j} \nabla_{j} \nabla_{i} f & =\nabla_{j}\left(\lambda g_{i j}+\rho R g_{i j}-R_{i j}\right) \\
& =\nabla_{i}\left(\rho R-\frac{1}{2} R\right)
\end{aligned}
$$

and

$$
\nabla_{i} \Delta f=\nabla_{i}(\lambda n+\rho R n-R)=\nabla_{i}(\rho R n-R)
$$

Therefore, (5) yields

$$
(n-1) \rho \nabla_{i} R-\frac{1}{2} \nabla_{i} R+R_{i j} \nabla_{j} f=0,
$$

Taking covariant derivative $\nabla_{l}$, we get

$$
(n-1) \rho \nabla_{l} \nabla_{i} R-\frac{1}{2} \nabla_{l} \nabla_{i} R+\nabla_{l} R_{i j} \nabla_{j} f+R_{i j} \nabla_{l} \nabla_{j} f=0 .
$$

Taking trace in both sides, we obtain

$$
\left((n-1) \rho-\frac{1}{2}\right) \Delta R+\frac{1}{2} g(\nabla R, \nabla f)+R(\lambda n+\rho R n-R)=0 .
$$

Now integrating using divergence theorem we get

$$
\begin{aligned}
\int_{M} R(\lambda n+\rho R n-R) & =-\int_{M}\left((n-1) \rho-\frac{1}{2}\right) \Delta R-\frac{1}{2} \int_{M} g(\nabla R, \nabla f) \\
& =\frac{1}{2} \int_{M} R \Delta f=\frac{1}{2} \int_{M} R(\lambda n+\rho R n-R) .
\end{aligned}
$$

The above equation is true only if

$$
\int_{M} R(\lambda n+\rho R n-R)=0,
$$

which implies

$$
\int_{M} R\left(R+\frac{\lambda n}{n \rho-1}\right)=0
$$


Again integrating (4), we obtain

$$
\int_{M}\left(R+\frac{\lambda n}{n \rho-1}\right)=0
$$

Therefore, (9) and (10) together imply that

$$
\int_{M}\left(R+\frac{\lambda n}{n \rho-1}\right)^{2}=0 .
$$

Hence, $R=\lambda n /(1-\rho n)$. Then from Theorem 1.3 we can conclude our result.

Proof of the Theorem 1.6. From (4) we get

$$
(n \rho-1) R=\Delta f-\lambda n .
$$

Since $\lambda \geq 0$, the above equation implies that

$$
(n \rho-1) R \leq \Delta f
$$

Now, we consider the cut-off function, introduced in [6], $\varphi_{r} \in C_{0}^{2}(B(p, 2 r))$ for $r>0$ such that

$$
\begin{cases}0 \leq \varphi_{r} \leq 1 & \text { in } B(p, 2 r) \\ \varphi_{r}=1 & \text { in } B(p, r) \\ \left|\nabla \varphi_{r}\right|^{2} \leq \frac{C}{r^{2}} & \text { in } B(p, 2 r) \\ \Delta \varphi_{r} \leq \frac{C}{r^{2}} & \text { in } B(p, 2 r),\end{cases}
$$

where $C>0$ is a constant. Then for $r \rightarrow \infty$, we have $\Delta \varphi_{r}^{2} \rightarrow 0$ as $\Delta \varphi_{r}^{2} \leq \frac{C}{r^{2}}$. Then we calculate

$$
\begin{aligned}
(n \rho-1) \int_{M} R \varphi_{r}^{2} \leq \int_{M} \varphi_{r}^{2} \Delta f & =\int_{B(p, 2 r)-B(p, r)} f \Delta \varphi_{r}^{2} \\
& \leq \int_{B(p, 2 r)-B(p, r)} f \frac{C}{r^{2}} \rightarrow 0
\end{aligned}
$$

as $r \rightarrow \infty$. Hence, we obtain

$$
(n \rho-1) \lim _{r \rightarrow \infty} \int_{B(p, r)} R \leq 0 .
$$

Since $\rho>1 / n$, it follows that

$$
\lim _{r \rightarrow \infty} \int_{B(p, r)} R \leq 0 .
$$

But $R$ is non-negative everywhere in $M$. Therefore, $R \equiv 0$ in $M$.

\section{ACKNOWLEDGMENT}

The third author gratefully acknowledges to the CSIR(File No.:09/025(0282)/2019-EMR-I), Govt. of India for financial assistance. 
A. A. SHAIKH, C. K. MONDAL, P. MANDAL

\section{REFERENCES}

[1] Bourguignon, J. P., Ricci curvature and Einstein metrics. Global differential geometry and global analysis, Berlin, 1979, 42-63, Lecture Notes in Math. 838, Springer, Berlin, 1981.

[2] Cao, H. D., Recent progress on Ricci solitons, in: Recent Advances in Geometric Analysis, Adv. Lectures Math., 11 (2010), 1-38.

[3] Cao, H. D. and Zhou, D., On complee gradient shrnking Ricci solitons. J. Diff. Geom., 85 (2010), 175-183.

[4] Catino, G. and Mazzieri, L., Gradient Einstein solitons, Nonlinear Anal., 132 (2016), 66-94.

[5] Catino, G., Mazzieri, L. and Mongodi, S., Rigidity of gradient Einstein shrinkers, Commun. Contemp. Math., 17(6) (2015), 1-18.

[6] Cheeger, J. and Colding, T. H., Lower bounds on Ricci curvature and the almost rigidity of warped products, Ann. Math., 144(1) (1996), 189-237.

[7] Chow, B. and Knopf, D., The Ricci flow: An introduction, mathematical surveys and monographs. Amer. Math. Soc., 110, 2004.

[8] Fang, F. Q., Man, J. W. and Zhang, Z. L., Complete gradient shrinking Ricci solitons have finite topological type. C. R. Acad. Sci. Paris, Ser. I 346(1971), 653-656.

[9] Hamilton, R. S., Three-manifolds with positive Ricci curvature, J. Differ. Geom., 17 (1982), 255-306.

[10] Huang, G., Integral pinched gradient shrinking $\rho$-Einstein solitons, J. Math. Ann. Appl. 451(2) (2017), $1045-1055$.

[11] $\mathrm{Hu}, \mathrm{Z} ., \mathrm{Li}, \mathrm{D}$. and Xu, J., On generalized m-quasi-Einstein manifolds with constant scalar curvature, J. Math. Ann. Appl. 432(2) (2015), 733-743.

[12] Huang, G. and Wei, Y., The classification of ( $m$, )-quasi-Einstein manifolds, Ann. Glob. Anal. geom. 44 (2013), 269-282.

[13] Dwivedi, S., Some results on Ricci-Bourguignon and Ricci-Bourguignon almost solitons, arXiv:1809.11103.

[14] Mondal, C. K. and Shaikh, A. A., Some results on $\eta$-Ricci Soliton and gradient rho-Einstein soliton in a complete Riemannian manifold, Comm. Korean Math. Soc. 34(4) (2019), 1279-1287.

Department of Mathematics,

University of Burdwan, Golapbag,

BURDWAN-713104,

West Bengal, India

E-mail address: aask2003@yahoo.co.in, aashaikh@math.buruniv.ac.in

E-mail address: chan.alge@gmail.com

E-mail address: prosenjitmandal235@gmail.com 